\title{
Cell cycle arrest biomarkers for predicting renal recovery from acute kidney injury: a prospective validation study
}

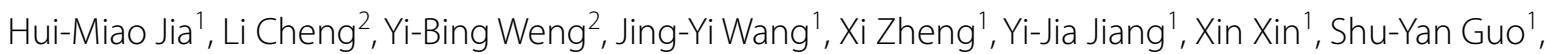
Chao-Dong Chen ${ }^{1}$, Fang-Xing Guo ${ }^{1}$, Yu-Zhen Han' ${ }^{1}$, Tian-En Zhang ${ }^{3}$ and Wen-Xiong Li*

\begin{abstract}
Background: Acute kidney injury (AKI) is a common disease in the intensive care unit (ICU). AKI patients with nonrecovery of renal function have a markedly increased risk of death compared with patients with recovery. The current study aimed to explore and validate the utility of urinary cell cycle arrest biomarkers for predicting nonrecovery in patients who developed AKI after ICU admission.
\end{abstract}

Methods: We prospectively and consecutively enrolled 379 critically ill patients who developed AKI after admission to the ICU, which were divided into a derivation cohort (194 AKI patients) and a validation cohort (185 AKI patients). The biomarkers of urinary tissue inhibitor of metalloproteinase-2 (TIMP-2) and insulin-like growth factor-binding protein 7 (IGFBP7) were detected at inclusion immediately after AKI diagnosis (day 0) and $24 \mathrm{~h}$ later (day 1). The optimal cut-off values of these biomarkers for predicting nonrecovery were estimated in the derivation cohort, and their predictive accuracy was assessed in the validation cohort. The primary endpoint was nonrecovery from AKI (within 7 days).

Results: Of 379 patients, 159 (41.9\%) patients failed to recover from AKI onset, with 79 in the derivation cohort and 80 in the validation cohort. Urinary [TIMP-2]* [IGFBP7] on day 0 showed a better prediction ability for nonrecovery than TIMP-2 and IGFBP7 alone, with an area under the reciever operating characteristic curve (AUC) of 0.751 [95\% confidence interval (Cl) 0.701-0.852, $p<0.001]$ and an optimal cut-off value of $1.05\left((\mathrm{ng} / \mathrm{mL})^{2} / 1000\right)$. When [TIMP$2]^{*}[\mathrm{IGFBP7]}$ was combined with the clinical factors of AKI diagnosed by the urine output (UO) criteria, AKI stage 2-3 and nonrenal SOFA score for predicting nonrecovery, the AUC was significantly improved to $0.852(95 \% \mathrm{Cl} 0.750-$ $0.891, p<0.001)$, which achieved a sensitivity and specificity of $88.8 \%(72.9,98.7)$ and $92.6 \%(80.8,100.0)$, respectively. However, urine [TIMP-2]*[IGFBP7], TIMP-2 alone, and IGFBP7 alone on day 1 performed poorly for predicting AKI recovery.

Conclusion: Urinary [TIMP-2]*[IGFBP7] on day 0 showed a fair performance for predicting nonrecovery from AKI. The predictive accuracy can be improved when urinary [TIMP-2]* [IGFBP7] is combined with the clinical factors of AKI diagnosed by the UO criteria, AKI stage 2-3 and nonrenal SOFA score.

Keywords: TIMP-2, IGFBP7, Acute kidney injury, Renal recovery, Prognosis

*Correspondence: liwx1126@163.com

${ }^{1}$ Department of Surgical Intensive Critical Unit, Beijing Chao-yang Hospital, Capital Medical University, 8 Gongren Tiyuchang Nanlu, Chaoyang District, Beijing 100020, China

Full list of author information is available at the end of the article

\section{Background}

Acute kidney injury (AKI) is a common disease in the intensive care unit (ICU) and carries a significant risk of chronic kidney disease (CKD) and short- and longterm mortality $[1-3]$. Currently, specific therapeutic 
interventions and available preventive measures are limited, so renal recovery after AKI has cumulatively become the focus of research. Moreover, changes in renal functional reserve may substantially affect the clinical outcomes of AKI patients [4-6]. AKI patients with nonrecovery of renal function have a markedly increased risk of death compared with recovery patients [7]. Therefore, preventing the nonrecovery of renal function should be the therapeutic goal of AKI.

Among all AKI biomarkers, cell cycle arrest of urinary tissue inhibitor of metalloproteinase-2 (TIMP-2) and insulin-like growth factor-binding protein 7 (IGFBP7) is upregulated early after AKI onset and has been confirmed to be superior in the early detection of AKI [8]. However, only a few studies have assessed their performance as prognostic markers for nonrenal recovery [5, 9]. If we can predict patients who will fail to recover in early AKI, effective supportive measures (for example, removal of nephrotoxic agents, optimization of volume management and individualized haemodynamic resuscitation) may be implemented early before irreversible recovery occurs $[10,11]$, which may prevent further progression of AKI and improve clinical prognosis. The current study, measuring urinary TIMP-2 and IGFBP7 when AKI was diagnosed, evaluated and validated the utility of urinary [TIMP-2]"[IGFBP7] for predicting nonrecovery in patients who developed AKI after ICU admission.

\section{Methods}

The study was approved by the Human Ethics Committee of Beijing Chao-yang Hospital, Capital Medical University (Beijing, China), and the ethics number was 2018-117. Written informed consent was obtained before patients were enrolled in this study.

\section{Study setting and population}

The present study was performed in two Chinese ICUs of Beijing Chao-yang Hospital and Beijing Lu-he Hospital from July 1, 2018, to December 1, 2020. The study design, performance, and report complied with the Standards for Reporting of Diagnostic Accuracy guidelines [12]. We critically screened patients who stayed in the ICU longer than $24 \mathrm{~h}$. Patients who developed AKI after ICU admission were prospectively and consecutively enrolled. The exclusion criteria were as follows: (1) age $<18$ years; (2) developed AKI before ICU admission; and (3) acquired insufficient urine samples at enrolment. All enrolled patients adhered to the following management principles: Kidney Disease: Improving Global Outcomes (KDIGO) bundle of optimization of volume status and haemodynamics, avoidance of nephrotoxic drugs, and prevention of hyperglycaemia [11]; active treatment of primary disease and comorbidities; the same principles of treatment with antibiotics; nutritional metabolism and organ support.

\section{Biomarker measurements}

Urine samples for biomarker assessment were taken from the urinary catheter of eligible patients soon after AKI was diagnosed and $24 \mathrm{~h}$ later. The biomarkers TIMP-2 and IGFBP7 were detected at inclusion (day 0 ) and $24 \mathrm{~h}$ later (day 1) and measured with the NephroCheck ${ }^{\mathrm{TM}}$ Test and VITROS 5600 Integrated System (Astute Medical, San Diego, CA, USA). The VITROS 5600 Integrated System reports the product of the two protein concentrations ([TIMP-2].[IGFBP7]) in units of $(\mathrm{ng} / \mathrm{mL})^{2} / 1000$. The biomarkers were measured by technicians who were blinded to the clinical data, and the physicians in charge were blinded to the biomarker test results.

\section{Clinical endpoint and definitions}

The diagnosis of AKI was dependent on the serum creatinine and urine output (UO) criteria proposed by the KDIGO as any of the following: increase in serum creatinine by $\geqq 0.3 \mathrm{mg} / \mathrm{dl}(\geqq 26.5 \mu \mathrm{mol} / \mathrm{L})$ within $48 \mathrm{~h}$; increase in serum creatinine to $\geqq 1.5$ times baseline; or $\mathrm{UO}<0.5 \mathrm{ml} / \mathrm{kg} / \mathrm{h}$ for $>6 \mathrm{~h}[13,14]$. The primary endpoint was nonrecovery from AKI. Renal recovery was defined as the absence of any stage of AKI by either serum creatinine criteria or UO criteria. For example, a patient with stage 2 AKI would have to have a decrease in serum creatinine to less than $150 \%$ of baseline and be free of periods of oliguria ( $\mathrm{UO}<0.5 \mathrm{ml} / \mathrm{kg} / \mathrm{h}$ ) for longer than $6 \mathrm{~h}$ [4]. Patients requiring renal replacement therapy (RRT) until the 7th day after AKI or who died within 7 days were regarded as nonrecovery patients. The secondary endpoints were the use of RRT in the ICU period, hospital mortality and 30-day mortality. AKI diagnosed by the UO criteria included patients who were diagnosed with AKI by the UO criteria alone or by both the UO and creatinine criteria. The baseline creatinine level was defined as follows: if at least five values were available, the median of all values available from 6 months to 7 days prior to enrolment was used. Otherwise, the lowest value in the 7 days prior to enrolment was used. If no pre-enrolment creatinine was available or the emergency patient's serum creatinine was abnormal at the time of admission, the baseline creatinine level was estimated using the Modification of Diet in Renal Disease (MDRD) equation assuming that the baseline glomerular filtration rate (GFR) was $75 \mathrm{ml} / \mathrm{min}$ per $1.73 \mathrm{~m}^{2}$. CKD was defined according to the definition of the National Kidney Foundation as estimated GFR $<60 \mathrm{ml} / \mathrm{min} / 1.73 \mathrm{~m}^{2}$ for at least 3 months irrespective of the cause $[15,16]$. Kinetic GFR for $24 \mathrm{~h}$ was calculated by the formula [17]. Sepsis was defined as life-threatening organ dysfunction caused by a 
dysregulated host response to infection as recommended by The Third International Consensus Definitions for Sepsis and Septic Shock (Sepsis-3) [18].

\section{Data collection}

All clinical data were prospectively collected on the basis of case report forms. Clinical patient variables included patient demographic characteristics, prior health history, diagnosis, comorbidities, use of vasopressors, and mechanical ventilation. Serum creatinine was detected and recorded at ICU admission and every $12 \mathrm{~h}$ thereafter until the 7th day after AKI. UO was measured hourly from the urinary catheter in the ICU period. The Acute Physiology and Chronic Health Evaluation II (APACHE II) and Sequential Organ Failure Assessment (SOFA) scores were assessed on the day AKI was diagnosed. Furthermore, the use of RRT in the ICU period, duration of ICU stay, hospital stay, and death in the hospital and 30 days after AKI development were recorded.

\section{Study phases}

The study had 2 phases. Phase I (derivation cohort) was performed from July 1, 2018, to July 31, 2019. This cohort was used to estimate the cut-off value of urinary [TIMP-2]"[IGFBP7] that best distinguished patients who would fail to recover after AKI developed. Phase II (validation cohort) was performed from August 1, 2019, to December 1, 2020. The predictive accuracy of urinary [TIMP-2]"[IGFBP7] for nonrecovery was assessed in the validation cohort using the cut-off value previously estimated in the derivation cohort.

\section{Statistical analysis}

SPSS statistics 24 (IBM, Chicago, IL) and R 2.1.2 were used for statistical analyses. Continuous variables are presented as the mean \pm standard deviation (SD) or the median (25th and 75th percentiles), and categorical variables are presented as percentiles. Continuous data were compared between two groups (recovery group and nonrecovery group) using repeated measurement analysis of variance or Mann-Whitney U tests, and categorical variables were compared using the Chi-square test or Fisher's exact test. For all analyses, statistical significance was indicated by a two-sided $p<0.05$.

In the derivation cohort, clinical parameters were compared between the two groups of patients with and without recovery. Clinical parameters with $p<0.15$ in univariate analyses were included in the multivariate logistic regression model. Variables with $p<0.05$ in the multivariate logistic regression model were independent risk factors for nonrecovery. A receiver operating characteristic (ROC) curve was used to assess the predictive values. The following values were used to describe the area under the ROC curve (AUC): $0.90-1.0$, excellent; $0.80-0.89$, good; $0.70-0.79$, fair; $0.60-0.69$, poor; and $0.50-0.59$, no useful performance. The optimal cut-off value was determined by the Youden index. The net contribution of the biomarkers to predict nonrecovery was validated by the Hosmer-Lemeshow test, net reclassification improvement (NRI) and integrated discrimination improvement (IDI). The DeLong test was used to compare the significant differences between the two AUCs.

In the validation cohort, the predictive accuracy of the biomarkers was assessed by sensitivity, specificity, positive predictive value (PPV), and negative predictive value (NPV).

\section{Results}

\section{Overall patient characteristics}

During the study period, 3154 critically ill patients who stayed longer than $24 \mathrm{~h}$ after ICU admission were screened in two ICUs; among them, 424 (13.4\%) patients developed AKI. After excluding the ineligible patients, 379 were finally enrolled, with 194 in the derivation cohort and 185 in the validation cohort. Baseline characteristics, comorbidities, AKI classification, and short-term prognosis showed no significant differences between the two cohorts. The comparisons are presented in Table 1. The flow diagram is shown in Fig. 1. Urinary TIMP-2 and IGFBP7 on day 0 were obtained from all 379 patients. Among the 379 patients, 17 (4.5\%) patients were lost from day 0 to day 1 because of anuria with or without RRT, and 11 patients (2.9\%) died within $24 \mathrm{~h}$.

\section{Characteristics and outcomes of AKI patients} with and without renal recovery in the derivation cohort

In the derivation cohort, 115 (59.3\%) patients had renal recovery from AKI onset, and 79 (40.7\%) patients suffered from nonrecovery. There were no significant differences in demographic characteristics or comorbidities between patients with and without renal recovery. However, the APACHE II score and nonrenal SOFA score were remarkably higher in patients who failed to recover than in patients who recovered. Moreover, $\mathrm{PaO}_{2} / \mathrm{FiO}_{2}$, use of vasopressors, AKI diagnosed by the UO criteria, serum creatinine diagnosis of AKI, kinetic GFR for $24 \mathrm{~h}$, persistent AKI and AKI stage 2-3 showed significant differences between patients with and without renal recovery. AKI diagnosed by the UO criteria, AKI stage 2-3, APACHE II score and nonrenal SOFA score were independent risk factors for nonrecovery of renal function in multivariate logistic regression. Significant differences in the biomarker concentrations of urinary [TIMP-2]"[IGFBP7], TIMP-2 and IGFBP7 on day 0 were observed. Recovery patients showed concentrations of $0.3(0.1,0.7)\left[(\mathrm{ng} / \mathrm{mL})^{2} / 1000\right], 3.3(2.2,6.3) \mathrm{ng} / \mathrm{mL}$, and 
Table 1 Patient baseline characteristics in derivation and validation cohorts

\begin{tabular}{|c|c|c|c|}
\hline Variables & Derivation cohort $(n=194)$ & Validation cohort $(n=185)$ & $p$ value \\
\hline \multicolumn{4}{|l|}{ Baseline characteristics } \\
\hline Age (year) & $61(50,71)$ & $60(49,74)$ & 0.773 \\
\hline Female gender & $117(60.3)$ & $122(65.9)$ & 0.239 \\
\hline $\mathrm{BMI}\left(\mathrm{kg} / \mathrm{m}^{2}\right)$ & $19.9(17.3,21.3)$ & $20.7(17.7,22.1)$ & 0.603 \\
\hline APACHE II score & $15(10,18)$ & $14(10,18)$ & 0.228 \\
\hline Nonrenal SOFA score & $5(1,8)$ & $5(2,8)$ & 0.543 \\
\hline Baseline serum creatinine $(\mu \mathrm{mol} / \mathrm{L})$ & $63.8(53.5,73.3)$ & $65.4(50.4,73.2)$ & 0.617 \\
\hline \multicolumn{4}{|l|}{ Admission type } \\
\hline Medical & $30(15.5)$ & $27(14.6)$ & 0.732 \\
\hline Surgical (abdominal surgery) & $93(47.8)$ & $86(46.5)$ & 0.892 \\
\hline Surgical (non-abdominal surgery) & $55(28.4)$ & $52(28.1)$ & 0.945 \\
\hline Emergency & $16(8.3)$ & $20(10.8)$ & 0.634 \\
\hline \multicolumn{4}{|l|}{ Main reason for admission } \\
\hline Respiratory system & $59(30.4)$ & $58(32.4)$ & 0.624 \\
\hline Circulatory system & $65(33.5)$ & $59(31.9)$ & 0.710 \\
\hline Digestive system & $42(21.6)$ & $43(23.2)$ & 0.547 \\
\hline Urinary system & $17(8.8)$ & $17(9.2)$ & 0.812 \\
\hline Others & $11(5.7)$ & $8(4.3)$ & 0.634 \\
\hline \multicolumn{4}{|l|}{ Comorbidities } \\
\hline COPD/asthma & $20(10.3)$ & $20(10.8)$ & 0.869 \\
\hline Cardiovascular disease & $40(20.6)$ & $43(23.2)$ & 0.536 \\
\hline Chronic liver disease & $42(21.6)$ & $41(22.2)$ & 0.902 \\
\hline Diabetes & $49(25.2)$ & $40(21.6)$ & 0.467 \\
\hline Hypertension & $91(46.9)$ & $79(42.7)$ & 0.469 \\
\hline CKD & $10(5.1)$ & $7(3.9)$ & 0.623 \\
\hline Sepsis & $89(45.8)$ & $69(37.3)$ & 0.102 \\
\hline Mechanical ventilation & $160(82.5)$ & $151(81.6)$ & 0.894 \\
\hline $\mathrm{PaO}_{2} / \mathrm{FiO}_{2}$ & $308.3(232.5,405.5)$ & $316.0(216.0,403.3)$ & 0.433 \\
\hline Use of vasopressor & $68(35.1)$ & $61(33.0)$ & 0.658 \\
\hline Use of diuresis & $28(14.4)$ & $23(12.4)$ & 0.724 \\
\hline AKI diagnosed by UO criteria & $65(33.5)$ & $64(34.6)$ & 0.812 \\
\hline AKI diagnosed by SCr criteria & $129(66.5)$ & $121(65.4)$ & 0.845 \\
\hline Serum creatinine diagnosing AKI ( $\mu \mathrm{mol} / \mathrm{L})$ & $128.6(86.2,173.4)$ & $132.5(90.4,178.6)$ & 0.415 \\
\hline Peak serum creatinine $(\mu \mathrm{mol} / \mathrm{L})$ & $273.5(154.6,412.3)$ & $292.3(174.6,432.5)$ & 0.228 \\
\hline \multicolumn{4}{|l|}{ AKI classification } \\
\hline Stage 1 & $104(53.6)$ & $94(38.7)$ & 0.588 \\
\hline 2 & $58(29.9)$ & $58(44.0)$ & 0.526 \\
\hline 3 & $32(16.5)$ & $33(17.8)$ & 0.713 \\
\hline \multicolumn{4}{|l|}{ Outcomes } \\
\hline Renal recovery in 7 days & $115(59.3)$ & $105(56.8)$ & 0.677 \\
\hline Renal recovery at hospital discharge & $112(57.7)$ & $103(55.7)$ & 0.683 \\
\hline Need of RRT in ICU & $35(18.1)$ & $30(19.3)$ & 0.583 \\
\hline Hospital mortality & $33(9.2)$ & $33(8.6)$ & 0.893 \\
\hline 30-day mortality & $34(13.3)$ & $42(12.9)$ & 0.393 \\
\hline
\end{tabular}

Values are median (interquartile range) or $\mathrm{n}(\%)$. AKI diagnosed by UO criteria was defined as patients who were diagnosed by UO criteria and/or SCr criteria, meanwhile AKI classification by UO criteria was equal or greater than $\mathrm{SCr}$ criteria. Otherwise, AKI was diagnosed by $\mathrm{SCr}$ criteria. Persistent AKI was defined as kidney dysfunction without recovery within $48 \mathrm{~h}$

AKI acute kidney injury, BMI body mass index, APACHE II Acute Physiology and Chronic Health Evaluation, SOFA Sequential Of Organ Failure Assessment, CKD chronic kidney disease, $U O$ urine output, $R R T$ renal replacement therapy 


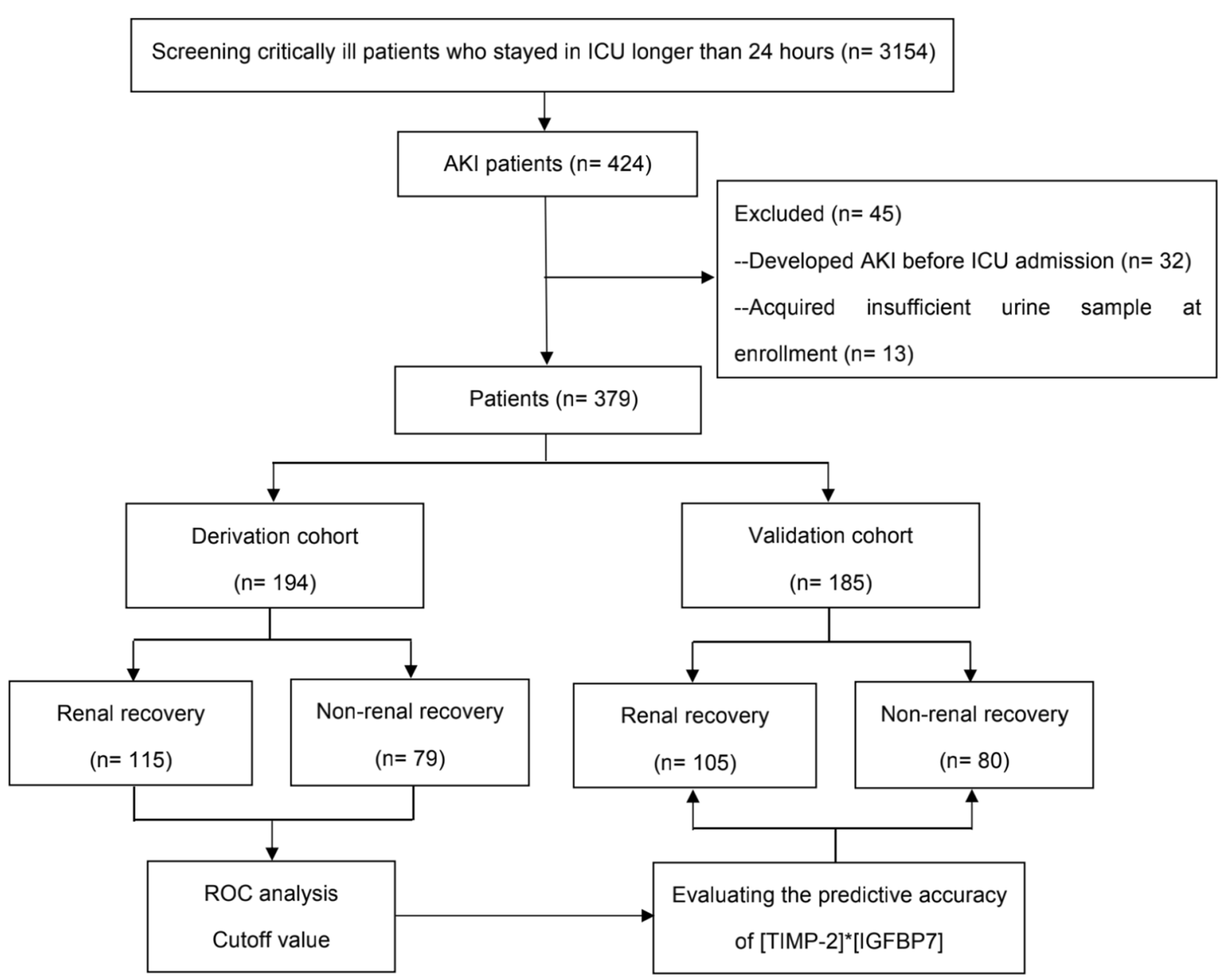

Fig. 1 Study flow diagram. AKI acute kidney injury, ICU intensive care unit, ROC receiver operating characteristic

$35.2(20.0,90.0) \mathrm{ng} / \mathrm{mL}$, respectively. However, patients who failed to recover showed higher concentrations of $1.1(0.3,5.5)\left[(\mathrm{ng} / \mathrm{mL})^{2} / 1000\right], 8.5(3.5,21.5) \mathrm{ng} / \mathrm{mL}$ and $100.9(41.2,329.1) \mathrm{ng} / \mathrm{mL}$, respectively. Table 2 summarizes these characteristic comparisons of patients with and without renal recovery.

RRT was used in 4 (3.4) recovery patients and 31 (39.2) nonrecovery patients. The duration of hospital stay was 24 (11.5-33.0) days in nonrecovery patients, which was longer than that in recovery patients [18 (11.5-25.0) days, $p=0.026$ ]. Moreover, 30-day mortality was higher in nonrecovery patients than in recovery patients [24 (30.3\%) vs. 19 (16.5\%), $p=0.018$ ]. Table 3 shows the outcome comparisons.

\section{Predicting nonrecovery from AKI in the derivation cohort}

AKI diagnosed by the UO criteria, AKI stage 2-3, APACHE II score and nonrenal SOFA score were independent risk factors for nonrecovery. There was a positive linear correlation between the APACHE II score and the nonrenal SOFA score $(r=0.567, p<0.001)$. Therefore, the nonrenal SOFA score, which had better predictive value, was included in the clinical risk prediction model. We used any two of the factors and all three factors to construct clinical models for comparisons to find the best prediction model. The clinical risk prediction model consisting of UO criteria, AKI stage 2-3, and nonrenal SOFA score achieved the best AUC of 0.722 [95\% confidence interval (CI) $0.640-0.802, p<0.001]$ for predicting nonrecovery from AKI.

Urinary [TIMP-2]"[IGFBP7] on day 0 showed an AUC of 0.751 (95\% CI $0.701-0.852, p<0.001$ ) for predicting nonrecovery from AKI with an optimal cutoff value of $1.05\left[(\mathrm{ng} / \mathrm{mL})^{2} / 1000\right]$. Moreover, TIMP-2 and IGFBP7 alone on day 0 also showed fair predictive value for nonrecovery, with AUCs of 0.744 (95\% CI $0.688-0.850, p<0.001)$ and 0.721 (95\% CI $0.537-0.806$, $p=0.037)$, respectively. However, the biomarkers urinary 
Table 2 Baseline characteristics between AKI patients with and without renal recovery in the derivation cohort

\begin{tabular}{|c|c|c|c|}
\hline Variables & Recovery $(n=115)$ & Non-recovery $(n=79)$ & $p$ value \\
\hline \multicolumn{4}{|l|}{ Baseline characteristics } \\
\hline Age (year) & $62(49,76)$ & $62(50,71)$ & 0.483 \\
\hline Female gender & $71(61.7)$ & $46(58.2)$ & 0.656 \\
\hline $\mathrm{BMI}\left(\mathrm{kg} / \mathrm{m}^{2}\right)$ & $23.3(20.5,24.8)$ & $22.6(19.5,23.9)$ & 0.354 \\
\hline APACHE II score & $14.0(12.0,16.0)$ & $16.0(14.0,18.0)$ & $<0.001$ \\
\hline Nonrenal SOFA score & $4(1,6)$ & $6(3,9)$ & $<0.001$ \\
\hline \multicolumn{4}{|l|}{ Admission type } \\
\hline Medical & $18(15.6)$ & $12(15.2)$ & 0.887 \\
\hline Surgical (abdominal surgery) & $51(44.3)$ & $42(53.2)$ & 0.213 \\
\hline Surgical (non-abdominal surgery) & $38(33.0)$ & $17(21.5)$ & 0.189 \\
\hline Emergency & $8(7.0)$ & $8(10.1)$ & 0.536 \\
\hline \multicolumn{4}{|l|}{ Comorbidities } \\
\hline COPD/asthma & $9(7.8)$ & $11(13.9)$ & 0.229 \\
\hline Cardiovascular disease & $26(22.6)$ & $14(17.7)$ & 0.472 \\
\hline Chronic liver disease & $26(22.6)$ & $16(20.3)$ & 0.726 \\
\hline Diabetes & $28(24.3)$ & $21(26.6)$ & 0.739 \\
\hline Hypertension & $50(43.5)$ & $41(51.9)$ & 0.305 \\
\hline CKD & $6(5.2)$ & $4(5.1)$ & 1.000 \\
\hline Sepsis & $51(44.3)$ & $38(48.1)$ & 0.661 \\
\hline Mechanical ventilation & $96(83.5)$ & $64(81.0)$ & 0.703 \\
\hline $\mathrm{PaO}_{2} / \mathrm{FiO}_{2}$ & $316.0(223.7,404.0)$ & $284.2(210.8,359.15)$ & 0.026 \\
\hline Use of vasopressor & $34(29.6)$ & $34(43.0)$ & 0.065 \\
\hline Use of diuresis & $13(11.3)$ & $15(19.0)$ & 0.216 \\
\hline AKI diagnosed by UO criteria & $30(26.1)$ & $35(44.3)$ & 0.002 \\
\hline Serum creatinine diagnosing AKI ( $\mu \mathrm{mol} / \mathrm{L})$ & $116.5(92.3,186.4)$ & $137.4(98.5,230.6)$ & $<0.001$ \\
\hline Kinetic GFR for $24 \mathrm{~h}\left(\mathrm{~mL} / \mathrm{min} / 1.73 \mathrm{~m}^{2}\right)$ & $55.4(40.2,86.3)$ & $38.4(19.8,58.7)$ & $<0.001$ \\
\hline AKI stage 2-3 & $39(33.9)$ & $51(64.5)$ & $<0.001$ \\
\hline Persistent AKI & $38(33.0)$ & $53(67.1)$ & $<0.001$ \\
\hline$\left[\right.$ TIMP-2]*[GFBP7] day $0\left[(\mathrm{ng} / \mathrm{mL})^{2} / 1000\right]$ & $0.3(0.1,0.6)$ & $1.1(0.2,5.5)$ & $<0.001$ \\
\hline TIMP-2 day 0 (ng/mL) & $3.3(2.2,6.3)$ & $8.5(3.5,21.5)$ & $<0.001$ \\
\hline IGFBP7 day 0 (ng/mL) & $35.2(20.0,90.0)$ & $100.9(41.2,329.1)$ & $<0.001$ \\
\hline [TIMP-2]*[IGFBP7] day $1\left[(\mathrm{ng} / \mathrm{mL})^{2} / 1000\right]$ & $0.3(0.1,0.8)$ & $0.4(0.2,1.8)$ & 0.104 \\
\hline TIMP-2 day 1 (ng/mL) & $3.6(2.5,6.8)$ & $5.9(2.8,13.5)$ & 0.591 \\
\hline IGFBP7 day 1 (ng/mL) & $70.0(40.6,120.4)$ & $73.2(30.7,179.5)$ & 0.019 \\
\hline
\end{tabular}

Values are median (interquartile range) or $n$ (\%). AKI diagnosed by UO criteria was defined as patients who were diagnosed by UO criteria and/or SCr criteria, meanwhile AKI classification by UO criteria was equal or greater than $\mathrm{SCr}$ criteria. Persistent AKI was defined as kidney dysfunction without recovery within $48 \mathrm{~h}$ AKl acute kidney injury, BMI body mass index, APACHE II Acute Physiology and Chronic Health Evaluation, SOFA Sequential Organ Failure Assessment, COPD chronic obstructive pulmonary disease, CKD chronic kidney disease, UO urine output, SCr serum creatinine, TIMP-2 tissue inhibitor of metalloproteinases-2, IGFBP-7 insulin-like growth factor-binding protein 7

[TIMP-2]*[IGFBP7], TIMP-2 and IGFBP7 on day 1 performed poorly for predicting nonrecovery. When [TIMP2]"[IGFBP7] on day 0 was combined with the clinical risk prediction model to predict nonrecovery, the power was significantly improved, resulting in the best predictive AUC of 0.852 (95\% CI 0.750-0.891, $p<0.001$ ), confirmed by the Hosmer-Lemeshow test $(p>0.05)$. The AUCs of the TIMP-2 day 0 and IGFBP7 day 0 clinical risk prediction models were 0.822 (95\% CI $0.744-0.900, p<0.001$ ) and 0.805 (95\% CI $0.725-0.886, p<0.001$ ), respectively.
The predictive value of the [TIMP-2]*[IGFBP7] day 0 clinical risk prediction model was superior to that of the TIMP-2 day 0 and IGFBP7 day 0 clinical risk prediction models in predicting nonrecovery from AKI, which was supported by the DeLong test, IDI and NRI (Additional file 1: Table S1).

Multivariate logistic regression analysis was used to calculate the probability of nonrecovery based on the [TIMP-2]*[IGFBP7] day 0 clinical risk prediction model: probability for nonrecovery $=1 /\left(1+\mathrm{e}^{-\mathrm{z}}\right)$, 
Table 3 Outcomes between AKI patients with and without renal recovery in the derivation cohort

\begin{tabular}{llll}
\hline Variables & Recovery $(\boldsymbol{n}=\mathbf{1 1 5})$ & $\begin{array}{l}\text { Non-recovery } \\
(\boldsymbol{n}=\mathbf{7 9})\end{array}$ & $\boldsymbol{p}$ value \\
\hline RRT & $4(3.4)$ & $31(39.2)$ & $<0.001$ \\
ICU stay (day) & $6(4,12)$ & $7.5(4,14)$ & 0.214 \\
Hospital stay (day) & $18(11.5,25)$ & $24(11.5,33)$ & 0.026 \\
Hospital mortality & $17(14.8)$ & $20(25.3)$ & 0.049 \\
30-day mortality & $19(16.5)$ & $24(30.3)$ & 0.018 \\
\hline
\end{tabular}

Values are median (interquartile range) or $n$ (\%)

$A K l$ acute kidney injury, ICU intensive care unit, $R R T$ renal replacement therapy

$\mathrm{z}=-2.451+0.397 *([$ TIMP-2 $] *[$ IGFBP7] day 0$)+0.060$

* nonrenal SOFA score +1.043 * AKI diagnosed by the UO criteria $+0.978 *$ AKI stage $2-3$. The optimal cutoff probability value was 0.290 . AKI patients who had a probability value greater than 0.290 may fail to recover. Table 4 shows the predictive performance outcomes of the biomarkers and combination models, and their ROC curves are presented in Fig. 2.

\section{Predictive accuracy of urinary [TIMP-2]*[IGFBP7]} for nonrecovery from AKI in the validation cohort In the validation cohort, 79/194 (40.7\%) patients failed to recover from AKI. The baseline characteristics of AKI patients with and without renal recovery in the validation cohort were compared (Additional file 1: Table S2). The predictive accuracy was assessed in the validation cohort using the cut-off values acquired in the derivation cohort. Urinary [TIMP-2]"[IGFBP7] on day 0 showed the best predictive accuracy for nonrecovery compared with urinary TIMP-2 and IGFBP7 alone, with sensitivity, specificity, PPV, and NPV values with 95\% CIs of $82.3 \%$ (67.4, 93.8), 76.9\% (72.4, 88.5), 65.0\% (43.2, 78.6) and
88.5\% $(76.3,95.8)$, respectively. When urinary [TIMP$2]^{*}[$ IGFBP7] on day 0 was combined with the clinical risk prediction model, the predictive accuracy was improved. The sensitivity, specificity, PPV and NPV increased to 88.8\% (72.9, 98.7), 86.2\% (70.4, 97.3), 80.0\% (65.9, 92.5) and $92.6 \%(80.8,100.0)$, respectively. The assessment of predictive accuracy for nonrecovery is shown in Table 5.

\section{Sensitivity analysis}

Of 379 patients, 198 (52.2\%) were diagnosed with stage 1 disease at enrolment, and $181(47.8 \%)$ were diagnosed with stage 2-3 disease. The patients were divided into two subgroups of stage 1 and stage $2-3$ according to the initial AKI stage. Predictive values were further assessed in the two subgroups. Urine [TIMP-2]*[IGFBP7] showed fair predictive value in patients with AKI stage 1 and stage 2-3 (Additional file 1: Table S3, S4).

\section{Discussion}

AKI remains a common and serious clinical syndrome in critically ill patients. It is well recognized that an episode of AKI may cause persistent impairment in renal function, with the potential to progress to CKD, the use of RRT and end-stage kidney disease (ESKD) with dialysis dependence, which is in turn strongly associated with increased short- and long-term mortality [3, 4]. Therefore, renal recovery after an episode of AKI is necessary. Urinary [TIMP-2]*[IGFBP7] was found to be useful for the risk stratification of patients at high risk of developing AKI [8]. The current study evaluated the ability of urinary [TIMP-2]*[IGFBP7] to predict failure to recover after AKI development. The main findings were as follows: (1) urine [TIMP-2]*[IGFBP7], TIMP-2 alone, and IGFBP7 alone on day 0 showed fair value for predicting nonrecovery from AKI. Urine [TIMP-2]*[IGFBP7] had the highest

Table 4 Biomarkers and combination models for predicting non-recovery from AKI

\begin{tabular}{|c|c|c|c|}
\hline & AUC $(95 \% \mathrm{Cl})$ & Cutoff value & $p$ value \\
\hline$\left[\right.$ TIMP-2]*[IGFBP7] day $0\left[(\mathrm{ng} / \mathrm{mL})^{2} / 1000\right]$ & $0.751(0.701,0.852)$ & 1.05 & $<0.001$ \\
\hline TIMP-2 day 0 (ng/mL) & $0.744(0.688,0.850)$ & 8.50 & $<0.001$ \\
\hline IGFBP7 day 0 (ng/mL) & $0.721(0.623,0.820)$ & 117.60 & $<0.001$ \\
\hline$\left[\right.$ TIMP-2]*[IGFBP7] day $1\left[(\mathrm{ng} / \mathrm{mL})^{2} / 1000\right]$ & $0.668(0.551,0.785)$ & 0.89 & 0.028 \\
\hline TIMP-2 day 1 (ng/mL) & $0.653(0.499,0.726)$ & 7.50 & 0.050 \\
\hline IGFBP7 day 1 (ng/mL) & $0.603(0.482,0.725)$ & 144.90 & 0.163 \\
\hline Clinical risk prediction model & $0.722(0.640,0.802)$ & 0.436 & $<0.001$ \\
\hline$\left([\mathrm{TIMP}-2]^{*}[\right.$ IGFBP7] day 0$)$ —clinical risk prediction model & $0.852(0.750,0.891)$ & 0.290 & $<0.001$ \\
\hline (TIMP-2 day 0)_clinical risk prediction model & $0.822(0.744,0.900)$ & 0.224 & $<0.001$ \\
\hline (IGFBP7 day 0)_clinical risk prediction model & $0.805(0.725,0.886)$ & 0.180 & $<0.001$ \\
\hline
\end{tabular}

Clinical risk prediction model consisting of AKI diagnosed by UO criteria, AKI stage 2-3 and nonrenal SOFA score

AUC area under the receiver operating characteristic, $C l$ confidence interval, AKI acute kidney injury, TIMP-2 tissue inhibitor of metalloproteinases-2, IGFBP-7 insulin-like growth factor-binding protein 7 

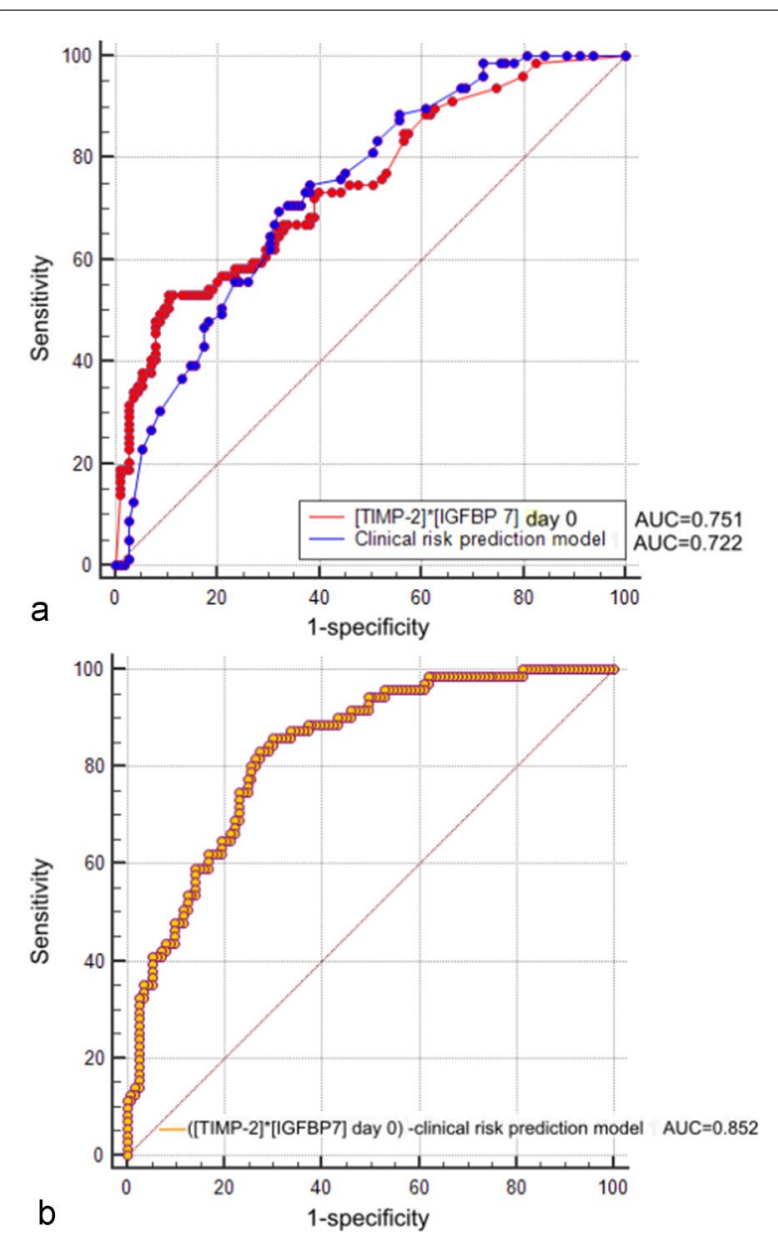

Fig. 2 Predictive value of biomarkers and the corresponding model. The ROC curves of urinary [TIMP-2]*[IGFBP7] on day 0 and the corresponding model for predicting failure to recover from AKI in the derivation cohort. a The AUCs of urinary [TIMP-2]*[IGFBP7] on day 0 and the clinical risk prediction model. $\mathbf{b}$ The AUC of the [TIMP-2]*[IGFBP7] day 0 clinical risk prediction model. $R O C$ receiver operating characteristic, AUC area under the ROC curve

AUC of 0.751 (95\% CI $0.701-0.852$ ), with a sensitivity and specificity of $82.3 \%$ and $76.9 \%$, respectively; (2) when adding urinary [TIMP-2]*[IGFBP7] on day 0 to the clinical risk prediction model, the predictive value was greatly improved to 0.852 . The utility of the [TIMP-2]"[IGFBP7] day 0 clinical risk prediction model was confirmed in the validation cohort, with a sensitivity and specificity of $88.8 \%$ and $92.6 \%$, respectively; and (3) urine [TIMP2]"[IGFBP7], TIMP-2 alone, and IGFBP7 alone on day 1 performed poorly for predicting AKI recovery.

Two novel biomarkers, urine TIMP-2 and IGFBP7, are inducers of G1 cell cycle arrest found in renal tubular cells. AKI is associated with the mechanisms of inflammation, oxidative stress, and apoptosis in cellular and molecular pathways $[19,20]$, and AKI may occur following ischaemic or toxic insults. TIMP-2 and IGFBP7 participate in these mechanisms and reflect early damage to the kidney [21].

The SAPPHIRE study first identified their ability to predict the development of KDIGO stage 2 or 3 AKI within $12 \mathrm{~h}$ in high-risk patients [8]. Many other studies subsequently confirmed their effective predictive value for the detection of AKI. Another endpoint of the SAPPHIRE study was the development of major adverse kidney events (MAKEs). The study showed that the risk of MAKEs increased sharply as the [TIMP-2]"[IGFBP7] level increased. It seems that a higher [TIMP2]*[IGFBP7] level was associated with worse outcomes. Another study also observed that higher median values of [TIMP-2]*[IGFBP7] were related to an increased degree of renal injury, and patients requiring RRT had the highest median [TIMP-2]*[IGFBP7] levels [22]. Dewitte A et al. [23] enrolled 57 consecutive patients presenting with AKI within the first $24 \mathrm{~h}$ after admission. They found that urinary [TIMP-2]"[IGFBP7] had a fair prediction ability for renal recovery within $48 \mathrm{~h}$ after AKI. If we could identify patients who would fail to recover renal function early, preventive measures and supportive treatments could be implemented early. Moreover, early recognition of nonrecovery can help physicians make decisions regarding fluid management and the timing of initiating RRT.

We enlarged the population by including consecutive AKI patients from two Chinese ICUs and used two cohorts to derive and validate the utility of urinary [TIMP-2]"[IGFBP7] for predicting patients who would fail to recover within 7 days. Urinary [TIMP-2]"IGFBP7] on day 0 showed a fair predictive value for nonrecovery. When it was added to a clinical risk prediction model

Table 5 Predictive accuracy of the biomarkers for non-recovery

\begin{tabular}{llllll}
\hline & Cutoff value & Sensitivity (\%) & Specificity (\%) & PPV (\%) & NPV (\%) \\
\hline$[\text { TIMP-2 }]^{*}\left[\right.$ IGFBP7] day 0 $\left[(\mathrm{ng} / \mathrm{mL})^{2} / 1000\right]$ & 1.05 & $82.3(67.4,93.8)$ & $76.9(72.4,88.5)$ & $65.0(43.2,78.6)$ & $88.5(76.3,95.8)$ \\
Clinical risk prediction model & 0.436 & $77.1(62.5,92.3)$ & $76.4(60.2,91.0)$ & $67.5(50.3,81.4)$ & $84.8(65.3,94.3)$ \\
{$[($ TIMP-2)*(IGFBP7) day 0]_Clinical risk } & 0.290 & $88.8(72.9,98.7)$ & $86.2(70.4,97.3)$ & $80.0(65.9,92.5)$ & $92.6(80.8,100.0)$
\end{tabular}


consisting of AKI diagnosed by the UO criteria, AKI stage 2-3 and nonrenal SOFA score, the performance for predicting nonrecovery improved. However, urinary [TIMP-2]*[IGFBP7] on day 1 performed poorly for predicting AKI recovery. The level of urinary [TIMP2]*[IGFBP7] in nonrecovery patients on day 1 was observed to be lower than that on day 0 , which may have led to the result that the prediction was fair on day 0 but poor on day 1. Furthermore, 28 (7.4\%) patients were lost because of anuria or death from day 0 to day 1 . Urinary [TIMP-2]"[IGFBP7] was not detected on day 1 in these patients. Therefore, the predictive result on day 1 may be affected.

In a previous study, many risk factors, such as age, comorbidity, more severe AKI, and severity of extrarenal organ dysfunction, were found to possibly contribute to nonrecovery after AKI $[5,13,23]$. Our study did not show a difference in age or comorbidity between patients with and without recovery, but more severe AKI and higher nonrenal SOFA scores were observed in nonrecovery patients. Notably, the variable of AKI diagnosed by the UO criteria played an important role in the prediction model for nonrecovery of AKI. In this study, 129 (34.0\%) patients showed oliguria (reaching the UO criteria for AKI diagnosis) and were diagnosed with AKI by UO. Oliguria is the oldest biomarker of AKI and may occur following a normal physiological response or reflect an underlying pathological process [24]. Many different pathophysiological pathways may cause oliguria, such as the neurohormonal pathway and absolute (hypovolemia) and relative (haemodynamic perturbations) reductions in effective blood volume [25]. Moreover, renal blood flow (RBF) may be preserved or even increased in sepsis-associated AKI. In this situation, abnormal distribution of intrarenal blood flow may be more influential than global RBF [26]. In addition to circulatory changes, immunologic and inflammatory mechanisms may participate in renal endothelial injury and microvascular dysfunction, which may lead to oliguria [26]. In a study by Federspiel CK et al. [27], $\mathrm{UO}<0.5 \mathrm{ml} / \mathrm{kg} / \mathrm{h}$ was associated with lower rates of resolving AKI (hazard ratio 0.31; 95\% CI 0.20-0.47). The usefulness of the combination of TIMP-2 and IGFBP7 with UO to improve risk stratification for severe outcomes was confirmed by the SAPPHIRE study [28]. Therefore, including the clinical factor of AKI diagnosed by the UO criteria for the prediction of renal recovery was reasonable.

Despite several meaningful findings, our study has several limitations. First, the included patients were critically ill patients from two ICU centres. The predictive value of urine [TIMP-2]*[IGFBP7] for renal recovery needs to be further assessed in a multicentre study with a larger sample size. Second, a high proportion of patients with stage
1 AKI were enrolled in the study. These patients were more likely to recover from AKI. Third, we tested the marker on day 0 and day 1 after AKI diagnosis. However, $7.4 \%$ of patients were lost to follow-up on day 1 , which may lead to bias in the statistical results. Further studies are still needed to evaluate the predictive power of serial measurements of urinary [TIMP-2]*[IGFBP7] for renal nonrecovery. Furthermore, we assessed short-term prognosis but did not explore long-term prognosis. It would also be helpful for the clinic to explore the association between urinary [TIMP-2]"[IGFBP7] and the long-term prognosis of AKI.

\section{Conclusion}

Urinary [TIMP-2]*[IGFBP7] on day 0 showed a fair performance for predicting failure to recover from AKI. The predictive accuracy can be improved when urinary [TIMP-2]*[IGFBP7] is combined with clinical factors of AKI diagnosed by the UO criteria, AKI stage $2-3$ and nonrenal SOFA score.

\begin{abstract}
Abbreviations
AKI: Acute kidney injury; ICU: Intensive care unit; KDIGO: Kidney disease: improving global outcomes; RRT: Renal replacement therapy; CKD: Chronic kidney disease; MDRD: Modification of diet in renal disease; SD: Standard deviation; Cl: Confidence interval; APACHE II: Acute Physiology and Chronic Health Evaluation; SOFA: Sequential Organ Failure Assessment; UO: Urine output; AUC: Area under the receiver operating characteristic curve; NRI: Net reclassification improvement; IDI: Integrated discrimination improvement; PPV: Positive predictive value; NPV: Negative predictive value.
\end{abstract}

\section{Supplementary Information}

The online version contains supplementary material available at https://doi. org/10.1186/s13613-022-00989-8.

Additional file 1: Table S1. DeLong test, NRI and IDI for assessing the contributions of different biomarkers for non-recovery prediction when combining with clinical model. Table S2. Baseline characteristics between AKI patients with and without renal recovery in the validation cohort. Table S3. Biomarkers for predicting non-recovery in patients with AKI stage 1. Table S4. Biomarkers for predicting non-recovery in patients with AKI stage 2-3.

Acknowledgements

We thank Professor Li-Rong Liang in Beijing Chao-yang Hospital for statistical analysis.

\section{Authors' contributions}

$\mathrm{H}-\mathrm{MJ}$ contributed to urine collection, data interpretation, drafting of the manuscript and critical revision of the manuscript. LC, Y-BE, XZ, J-YW and Y-J contributed to urine collection and data interpretation and performed statistical analysis. XX, S-YG, C-DC, F-XG and Y-ZH contributed to data collection and data interpretation. $\mathrm{W}-\mathrm{XL}$ chaired the group, conceived and designed the study, performed statistical analysis and contributed to data collection, data interpretation, and critical revision of the manuscript. All authors reviewed the manuscript. All authors read and approved the final manuscript.

\section{Funding}

This study is supported by Beijing Municipal Science \& Technology Commission (No. Z191100006619032, No. Z181100001718204). 
Availability of data and materials

All data generated and/or analysed during this study are included in this published article.

\section{Declarations}

\section{Ethics approval and consent to participate}

The study was approved by the Human Ethics Committee of Beijing ChaoYang Hospital, Capital Medical University (Beijing, China), the ethics number was 2018-117. Written informed consent was obtained before patients were enrolled in this study.

\section{Consent for publication}

The manuscript has been read and its submission approved by all co-authors.

\section{Competing interests}

The authors declare that they have no competing interests.

\section{Author details}

${ }^{1}$ Department of Surgical Intensive Critical Unit, Beijing Chao-yang Hospital, Capital Medical University, 8 Gongren Tiyuchang Nanlu, Chaoyang District, Beijing 100020, China. ${ }^{2}$ Department of Emergent Intensive Critical Unit, Beijing Lu-He Hospital, Capital Medical University, Beijing 101100, China. ${ }^{3}$ Gettysburg, PA 17325, USA

Received: 7 April 2021 Accepted: 29 January 2022

Published online: 12 February 2022

\section{References}

1. Bellomo R, Kellum JA, Ronco C. Acute kidney injury. Lancet. 2012;380:756-66.

2. Kaddourah A, Basu RK, Bagshaw SM, Goldstein SL, AWARE Investigators. Epidemiology of acute kidney injury in critically ill children and young adults. N Engl J Med. 2017;376:11-20.

3. Coca SG, Singanamala S, Parikh CR. Chronic kidney disease after acute kidney injury: a systematic review and meta-analysis. Kidney Int. 2012;81:442-8.

4. Kellum JA, Sileanu FE, Bihorac A, Hoste EA, Chawla LS. Recovery after acute kidney injury. Am J Respir Crit Care Med. 2017;195:784-91.

5. Forni LG, Darmon M, Ostermann M, Oudemans-van Straaten HM, Pettilä $\checkmark$, Prowle JR, et al. Renal recovery after acute kidney injury. Intensive Care Med. 2017;43:855-66.

6. Bellomo R, Ronco C, Mehta RL, Asfar P, Boisramé-Helms J, Darmon M, et al. Acute kidney injury in the ICU: from injury to recovery: reports from the 5th Paris international conference. Ann Intensive Care. 2017;7:49.

7. Cho WY, Lim SY, Yang JH, Oh SW, Kim MG, Jo SK. Urinary tissue inhibitor of metalloproteinase-2 and insulin-like growth factor-binding protein 7 as biomarkers of patients with established acute kidney injury. Korean $J$ Intern Med. 2020;35:662-71.

8. Kashani K, Al-Khafaji A, Ardiles T, Artigas A, Bagshaw SM, Bell M, et al. Discovery and validation of cell cycle arrest biomarkers in human acute kidney injury. Crit Care. 2013;17:R25.

9. Pickkers P, Ostermann M, Joannidis M, Zarbock A, Hoste E, Bellomo R, et al. The intensive care medicine agenda on acute kidney injury. Intensive Care Med. 2017:43:1198-209.

10. Ronco C. Acute kidney injury: from clinical to molecular diagnosis. Crit Care. 2016:20:201

11. Meersch M, Schmidt C, Hoffmeier A, Van Aken H, Wempe C, Gerss J, et al. Prevention of cardiac surgery-associated AKI by implementing the KDIGO guidelines in high risk patients identified by biomarkers: the PrevAKI randomized controlled trial. Intensive Care Med. 2017;43:1551-61.

12. Cohen JF, Korevaar DA, Altman DG, Bruns DE, Gatsonis CA, Hooft L, et al. STARD 2015 guidelines for reporting diagnostic accuracy studies: explanation and elaboration. BMJ Open. 2016;6:e012799.

13. Ostermann M, Joannidis M. Acute kidney injury 2016: diagnosis and diagnostic workup. Crit Care. 2016;20:299.

14. Chawla LS, Bellomo R, Bihorac A, Goldstein SL, Siew ED, Bagshaw SM, et al. Acute kidney disease and renal recovery: consensus report of the acute disease quality initiative (ADQI) 16 workgroup. Nat Rev Nephrol. 2017. https://doi.org/10.1038/nrneph.2017.2.

15. Helou R. Should we continue to use the Cockcroft-Gault formula? Nephron Clin Pract. 2010;116:c172-85.

16. Pilarczyk K, Edayadiyil-Dudasova M, Wendt D, Demircioglu E, Benedik J, Dohle DS, et al. Urinary [TIMP-2] ${ }^{*}$ [IGFBP7] for early prediction of acute kidney injury after coronary artery bypass surgery. Ann Intensive Care. 2015;5:50.

17. O'Sullivan ED, Doyle A. The clinical utility of kinetic glomerular filtration rate. Clin Kidney J. 2017;10:202-8.

18. Singer M, Deutschman CS, Seymour CW, Shankar-Hari M, Annane D, Bauer $M$, et al. The third international consensus definitions for sepsis and septic shock (Sepsis-3). JAMA. 2016;315:801-10.

19. Wang Z, Holthoff JH, Seely KA, Pathak E, Spencer HJ, Gokden N, et al. Development of oxidative stress in the peritubular capillary microenvironment mediates sepsis-induced renal microcirculatory failure and acute kidney injury. Am J Pathol. 2012;180:505-16.

20. Husi H, Human C. Molecular determinants of acute kidney injury. J Inj Violence Res. 2015;7:75-86.

21. Yang QH, Liu DW, Long Y, Liu HZ, Chai WZ, Wang XT. Acute renal failure during sepsis: potential role of cell cycle regulation. J Infect. 2009;58:459-64.

22. Gocze I, Koch M, Renner P, Zeman F, Graf BM, Dahlke MH, et al. Urinary biomarkers TIMP-2 and IGFBP7 early predict acute kidney injury after major surgery. PLoS ONE. 2015;10:e0120863.

23. Dewitte A, Joannès-Boyau O, Sidobre C, Fleureau C, Bats ML, Derache $P$, et al. Kinetic eGFR and novel AKI biomarkers to predict renal recovery. Clin J Am Soc Nephrol. 2015;10:1900-10.

24. Küllmar M, Meersch M. Intraoperative oliguria: physiological or beginning acute kidney injury? Anesth Analg. 2018;127:1109-10.

25. Klein SJ, Lehner GF, Forni LG, Joannidis M. Oliguria in critically ill patients: a narrative review. J Nephrol. 2018;31:855-62.

26. Fani F, Regolisti G, Delsante M, Cantaluppi V, Castellano G, Gesualdo L, et al. Recent advances in the pathogenetic mechanisms of sepsis-associated acute kidney injury. J Nephrol. 2018;31:351-9.

27. Federspiel CK, Itenov TS, Mehta K, Hsu RK, Bestle MH, Liu KD. Duration of acute kidney injury in critically ill patients. Ann Intensive Care. 2018;8:30.

28. Joannidis M, Forni LG, Haase M, Koyner J, Shi J, Kashani K, et al. Use of cell cycle arrest biomarkers in conjunction with classical markers of acute kidney injury. Crit Care Med. 2019. https://doi.org/10.1097/CCM.00000 00000003907.

\section{Publisher's Note}

Springer Nature remains neutral with regard to jurisdictional claims in published maps and institutional affiliations.

\section{Submit your manuscript to a SpringerOpen ${ }^{\circ}$ journal and benefit from:}

- Convenient online submission

$\checkmark$ Rigorous peer review

- Open access: articles freely available online

- High visibility within the field

- Retaining the copyright to your article

Submit your next manuscript at $>$ springeropen.com 